\title{
ESTUDIO DE LA INTELIGENCIA DE NEGOCIOS EN LAS REDES SOCIALES MÁS UTILIZADAS
}

Study of intelligence in business networks used more

\author{
Jaime Orozco ${ }^{1}$, Freddy Soriano ${ }^{2}$ \\ Escuela el Informática y Electrónica \\ ${ }^{1}$ Universidad Estatal Península de Santa Elena (UPSE) \\ Campus La Libertad, vía principal Santa Elena - La Libertad \\ La Libertad - Ecuador \\ jorozco@upse.edu.ec
}

\begin{abstract}
Resumen
El estudio en mención tiene como finalidad reconocer las redes sociales más utilizadas. La bibliografía anunciada en 20 citas entre opiniones, revistas libros, tesis, entre otros, que son conseguido con las siguientes palabras claves: "Business Intelligence", "Social Networks". La información encontrada entre el año 2008 al 2014 cuyas publicaciones dan a conocer que en el año 2013 se registraron más citas sobre este tema. La tesis de Noelia Ruiz Martínez la consideramos la primordial para este estudio, cuyo aportes muestra el análisis de la información en las redes sociales, como las herramientas que se utilizan. Este artículo aporta con información precisa y confiable sobre el uso de inteligencia de negocios.
\end{abstract}

Palabras clave: Inteligencia de Negocios, Redes Sociales.

\begin{abstract}
The study in question is intended to recognize the most used social networks. The bibliography announced 20 appointments in between reviews, magazines books, theses, among others, that are achieved with the following key words: "Business Intelligence", "Social Networks". The information found from 2008 to 2014 whose publications disclose that in 2013 more quotes on this subject were recorded. The thesis Noelia Ruiz Martínez consider it the primary for this study, whose contributions shows the analysis of information in social networks, such as the tools used. This brings articulates with accurate and reliable information on the use of business intelligence information.
\end{abstract}

Keyworks: Business Intelligence, Social Networks. 


\section{Introducción}

Las grandes protagonistas actuales de la sociedad digital son las redes sociales (David Caldevilla, 2010). Hoy en día ya no se cuestiona el inigualable poder que están adquiriendo y la importancia que tendrán, en poco tiempo, como generadoras de oportunidades, que quién sabe si trasformarán hacia nuevas y complejos grupos sociales que aún ni somos capaces de imaginar (David Caldevilla, 2010).

Cada vez se va incrementando, las empresas que desarrollan sus propias redes sociales con el ánimo de diferenciarse de sus rivales y proporcionar un valor agregado a sus clientes potenciales. Este hecho se debe a los beneficios que para las instituciones que descienden de la participación del consumidor en este tipo de redes. A modo de ejemplo, gracias a la aparición y al éxito de las redes sociales, se ha producido un gran auge del llamado marketing viral. Este marketing utiliza información procedente de medios como blogs, correos electrónicos y redes sociales con objeto de mejorar la posición de las empresas en el mercado.

Analizando la información transmitida en este "boca a boca" electrónico, las empresas y organizaciones pueden conocer información muy relevante y en tiempo real que les ayude en la toma de decisiones como, por ejemplo, la valoración de sus productos, las críticas recibidas, conocer quiénes son sus clientes fieles, el perfil de sus detractores y qué clientes son más influyentes así como compararse con sus competidores.

Otra de las ventajas que supone este tipo marketing es su costo muy bajo en asimilación con el habitual, así también reducen los costos de servicios técnicos, ya que los individuos que forman parte de estas redes se asisten mutuamente con el fin de resolver los problemas que aparecen en el uso de un producto o servicio Inteligencia de negocio (Casaló, Flavián, \& Guinaliú, 2009).

Así podemos encontrar las redes sociales más populares en este ranking que está basado en el análisis realizado por el sitio web Alexa, la cual es una subsidiaria de la empresa Amazon.com, la cual provee información sobre el tráfico de los sitios web a nivel global. (Culturiarte, 2013).

\begin{tabular}{|l|r|r|}
\hline Red Social & $\begin{array}{l}\text { Estimación de } \\
\text { visitantes } \\
\text { mensuales }\end{array}$ & Actualizado \\
\hline 1. Facebook & $\mathbf{7 5 0 0 0 0 0 0 0}$ & 01.10 .2014 \\
\hline 2. Twitter & $\mathbf{2 5 0 0 0 0 0 0 0}$ & 10.01 .2014 \\
\hline 3. LinkedIn & $\mathbf{1 1 0 0 0 0 0 0 0}$ & 01.10 .2014 \\
\hline 4. Pinterest & $\mathbf{8 5 5 0 0 0 0 0}$ & 01.10 .2014 \\
\hline 5. MySpace & $\mathbf{7 0 5 0 0 0 0 0}$ & 10.01 .2014 \\
\hline 6. Google Plus + & $\mathbf{6 5 0 0 0 0 0 0}$ & 01.10 .2014 \\
\hline 7. DeviantArt & $\mathbf{2 5 5 0 0 0 0 0}$ & 01.10 .2014 \\
\hline 8. LiveJournal & $\mathbf{2 0 5 0 0 0 0 0}$ & 10.01 .2014 \\
\hline 9. Tagged & $\mathbf{1 9 5 0 0 0 0 0}$ & 10.01 .2014 \\
\hline 10. Orkut & $\mathbf{1 7 5 0 0 0 0 0}$ & 01.10 .2014 \\
\hline 11. CafeMom & $\mathbf{1 2 5 0 0 0 0 0}$ & 01.10 .2014 \\
\hline 12. Ning & $\mathbf{1 2 0 0 0 0 0 0}$ & 01.10 .2014 \\
\hline 13. Meetup & $\mathbf{7 . 5 0 0 . 0 0 0}$ & 10.01 .2014 \\
\hline 14. MyLife & $\mathbf{5 . 4 0 0 . 0 0 0}$ & 10.01 .2014 \\
\hline 15. Ask.fm & $\mathbf{4 . 3 0 0 . 0 0 0}$ & 10.01 .2014 \\
\hline
\end{tabular}

Tabla 1: Redes sociales más populares

Fuente: (Amazon, 2014)

En el presente escenario, se ha realizado un la revisión de diversos artículos para entender la inteligencia de negocios. Existen diversos tipos de investigaciones, tanto teóricas como prácticas, en esta área, pero con enfoques más amplios.

\section{UTILIDADES DE LAS REDES SOCIALES}

El comienzo de nuevas plataformas de comunicación social, como son las redes sociales, en los años posteriores se ha puesto de manifiesto la capacidad de creación de las industrias de la información y el entretenimiento. La propagación precipitada de esta nueva manera de comunicarse y realizar nuevo contenido muy particular hace plantearse preguntas tales como hasta qué punto se está presenciando un cambio radical en el uso de medios de comunicación.

En este argumento, inversores provenientes tanto del sector del capital de riesgo como los medios de comunicación tradicionales y de otras organizaciones adyacentes han apostado por este novísimo fenómeno con una agresividad estratégica y financiera que nos hace recordar la fiebre del 'punto.com' de los 90. Analiza la dinámica subyacente en la difusión de estas redes sociales, su evolución futura (Raúl L. Katz, Phaedra Chrousos, Haley Wu, 2008)

La importancia de las redes sociales en la sociedad, ha efectuado un impacto, que los usuarios buscan un vínculo "sentimental" (Bidart, 2009), tal como se muestra la siguiente tabla del estudio: 


\begin{tabular}{|c|c|}
\hline $\begin{array}{c}\text { Aquello que los } \\
\text { vincula }\end{array}$ & $\%$ \\
\hline Vínculo familiar & 28.1 \\
\hline Lazo familiar & 25.2 \\
\hline Placer de estar juntos & 18.7 \\
\hline Amigos en común & 15.4 \\
\hline $\begin{array}{c}\text { Actividades en } \\
\text { común }\end{array}$ & 13.2 \\
\hline Pasado común & 12.6 \\
\hline Sus cualidades & 9.4 \\
\hline Se puede confiar & 6.6 \\
\hline No mucho & 2.1 \\
\hline Ayuda mutua & 3.7 \\
\hline Otro & 1.1 \\
\hline Nuestros hijos & 1.0 \\
\hline
\end{tabular}

Tabla 2: frecuencias de respuestas elegidas a un grupo de personas Fuente: (Bidart, 2009)

Esto nos permite el vínculo que se acepta el uso de internet para relacionar una nueva amistad. La aplicación de inteligencia de negocios en las redes sociales más utilizadas, el impacto será primordial en base al análisis de información que se va recolectar, procesar, y analizar para aplicar a la empresa. Es así que el cumplimiento de metas y objetivos de una organización se basara en la mejor decisión y el éxito de la misma. (Hsinchun Chen, Roger H. L. Chiang, Veda C. Storey, 2012)..

El uso de las redes sociales en lugares de trabajo tiende al individuo ser productivo o casi nada productivo, es por eso que las limitaciones del acceso a internet son muy comunes con el fin de salvaguardar la información de la empresa, pero si la organización es conocer las opiniones, expectativas, sugerencias de sus clientes, además de presentar nuevos productos de la empresa. El hecho de interactuar el cliente con la empresa se mejora los lazos entre ellos ("Opinión: El uso de las redes sociales en el trabajo Crhoy.com," n.d.).

Aunque algunos autores opinan y dicen que "No todos los negocios necesitan todas las redes ni todas las redes sirven para todos los negocios. Lo que sí es cierto es que son una herramienta que puede convertirse en el aliado de la compañía o negocio" ("Breve manual de redes sociales

para Pymes - Forbes México," n.d.). Dicha frase, ayuda entender la importancia de las redes sociales en la empresa siempre y cuando sea aplicado por mejorar. Buscar clientes en las redes sociales es difícil obtenerlos, pero si el cliente busca es mucho mejor porque ellos buscan lo que quieren.

El beneficio que pueda obtener las empresas aplicando $\mathrm{BI}$ en las redes sociales, según un estudio realizado en México, (Luis Enrique Valdez Juárez, Jesús Antonio Rascón Ruiz, Elva Alicia Ramos Esocbar, Jorge Enrique Huerta Gaxiola, 2012), el cual el correo electrónico es el más utilizado por los usuarios, el mismo que servirá para dar a conocer el servicio o producto, y a su vez dar posicionamiento.

\begin{tabular}{|c|c|c|c|c|}
\hline PLAN FACEBOOK & FB1 & FB2 & FB3 & FB4 \\
\hline PRECIO (\$USD) & $\$ 147$ & $\$ 247$ & \$ 397 & $\$ 497$ \\
\hline \multicolumn{5}{|l|}{ Características } \\
\hline $\begin{array}{l}\text { Página de } \\
\text { bienvenida/diseño en } \\
\text { torno a la temática del } \\
\text { texto. Hasta } 3 \text { enlaces } \\
\text { externos. }\end{array}$ & - & - & - & - \\
\hline $\begin{array}{l}\text { Imagen de perfil } \\
\text { personalizada/diseño del } \\
\text { banner de muro con su } \\
\text { logo o marca personal }\end{array}$ & - & - & - & - \\
\hline $\begin{array}{l}\text { Video o animación flash } \\
\text { integrada a la página } \\
\text { (entregada por el cliente) }\end{array}$ & & - & - & - \\
\hline $\begin{array}{l}\text { Micro web con hasta } 4 \\
\text { páginas internas de } \\
\text { contenido estático (texto, } \\
\text { imágenes, hasta } 3 \text { enlaces } \\
\text { c/u) }\end{array}$ & & & - & - \\
\hline $\begin{array}{l}\text { Formulario de contacto } \\
\text { con redirección a su } \\
\text { correo personal }\end{array}$ & & & & - \\
\hline $\begin{array}{l}\text { Diseño de cajas de } \\
\text { contenido con redirección } \\
\text { a su correo personal }\end{array}$ & & & & - \\
\hline $\begin{array}{l}\text { Integración en el diseño } \\
\text { de las últimas } \\
\text { actualizaciones de su } \\
\text { blog o sus tweets }\end{array}$ & & & & - \\
\hline $\begin{array}{l}\text { Galería de fotos integrada } \\
\text { en el diseño (se actualiza } \\
\text { automáticamente al subir } \\
\text { más fotos) }\end{array}$ & & & & - \\
\hline $\begin{array}{l}\text { Integración en el diseño } \\
\text { de caja de comentarios de } \\
\text { sus clientes/seguidores }\end{array}$ & & & & - \\
\hline
\end{tabular}

Tabla 3: Tablas de precios

Fuente:(Internet, 2013) 


\section{Objetivos del estudio}

Este estudio tiene los siguientes objetivos:

> Identificar las redes sociales más utilizadas.

$>$ Conocer las principales herramientas que brindan estas redes sociales como apoyo a la inteligencia de negocios.

> Analizar el costo beneficio que brindan las redes sociales a la inteligencia de negocios

\section{Alcance del estudio}

El presente documento contiene la revisión de artículos publicados que se encuentran en la base de datos de Mendeley y que entre ellos fueron incrementados con páginas web referentes al tema. Así también el mencionado estudio pretende identificar las redes sociales más utilizadas que aporten a la inteligencia de negocios, así como también revisar cómo invertir en estas redes sociales y a su vez esta nos ayuden a incrementar nuestro negocio.

\section{Materiales y métodos}

Se ha utilizado páginas web de conocimiento de $\mathrm{BI}$, redes sociales. Libros sobre avances tecnológicos en el sector automovilístico, como también diferentes artículos. La estrategia de investigación de información se la mostramos en el siguiente gráfico.

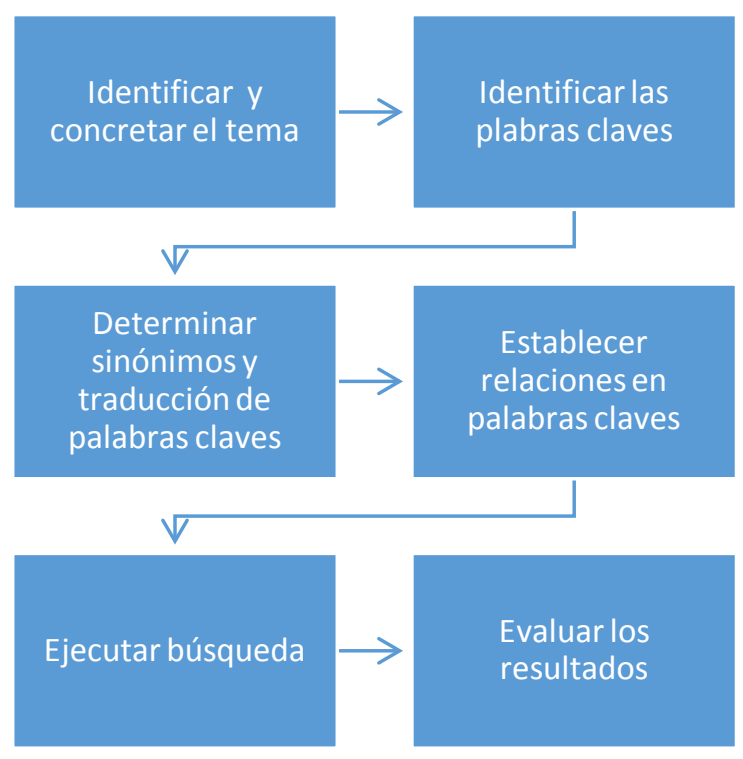

Grafico 2. Estrategia de investigación de información.

Fuente: (Richard Ramírez-Anormaliza1, Xavier Linàs-Audet2, 2013)

Las palabras claves que se utilizaron se las muestra en la siguiente tabla.

\begin{tabular}{|l|l|}
\hline Español & Ingles \\
\hline $\begin{array}{l}\text { Inteligencia de } \\
\text { negocios }\end{array}$ & $\begin{array}{l}\text { Business } \\
\text { intelligence }\end{array}$ \\
\hline Redes sociales & Social Networks \\
\hline
\end{tabular}

Para la colección de los datos bibliográficos, se utilizó el software Mendeley, el mismo que sirvió para nuestra bibliografía y por supuesto cumpliendo con los estándares APA (American Psychological Association)

\section{Análisis y discusión}

El uso de las redes sociales en lugares de trabajo tiende al individuo ser productivo o poco productivo, es por eso que las restricciones del acceso a internet son muy comunes con el fin de salvaguardar la información de la empresa, pero si la organización es conocer las opiniones, expectativas, sugerencias de sus clientes, además de presentar nuevos productos de la empresa. El hecho de interactuar el cliente con la empresa se mejora la relación entre ellos. (Moreira, 2014).

\section{Inteligencia de Negocios (Business Intelligence)}

Es un concepto que integra como solución el almacenamiento y procesamiento de enormes cantidades de datos e información para transformarla en conocimiento $y$ decisiones en tiempo real a través de una fácil explotación

\section{Inteligencia de negocios basada en tendencias de las redes sociales.}

Desde el inicio de las redes sociales, las empresas han pensado como utilizar toda la información que existe para su favor como parte de su campaña de Inteligencia de negocios. Esto puede ser una ventaja competitiva en tener información pública en tiempo real de sus competidores e industrias, analizar y comparar esta información puede llegar a ser muy complicado debido a la cantidad de información que es compartida segundo a segundo dentro de las redes sociales, (Elliott, 2004). 
Gracias a varias aplicaciones, los científicos de la información en internet, logran tener herramientas para extraer esta información y que las empresas puedan hacer las mejores decisiones para sus negocios.

Una de las herramientas que he estado utilizando y que ayuda a desarrollar análisis dentro de las redes sociales es InfiniGraph quienes cuentan con un proceso llamado Hypercuration.

La herramienta ayuda a identificar lo siguiente:

"Inteligencia en la información que existe en las redes sociales gracias a una colaboración de personas, ayuda a identificar una clasificación de cada uno de las publicaciones realizadas y a desarrollar tácticas para la interacción con el cliente ideal", (Elliott, 2004) Basado en una prueba gratuita, tuvimos la oportunidad de empezar a utilizar InfiniGraph, aquí tiene tres elementos de la aplicación, que le pueden ayudar si le interesa llevar al siguiente nivel su estrategia digital.

\section{Dashboard.}

Dentro del Dashboard, los usuarios pueden ver 3 componentes de la herramienta, que le ayuda a unificar parte de la inteligencia en redes sociales:

- Inteligencia de negocios dentro de los buscadores.

- Una lista de las industrias principales.

- Una lista de las marcas mejor posicionadas en redes sociales.

- Dependiendo de cómo desee seleccionar su información. InfiniGraph se podrá mostrar en distintos formatos.

En la época actual, el dinero sigue siendo el último factor de la popularidad de una empresa, la influencia, y la marca en los medios sociales? Ver ideas de compromiso y el contenido que está trabajando en las megas marcas aquí.

Hasta cierto punto sí, sin embargo, otro elemento aún más importante ha surgido en las prácticas de marketing de hoy en día. Content Marketing. La clara calidad del contenido tiene una enorme influencia en SEO (Search Engine Marketing Professional Organization), sobre la imagen de una empresa y de pie dentro de los medios de comunicación social. A través de su contenido, que también es su público, comprometidos, interesados y satisfechos para impulsar la empresa a convertirse en un líder dentro de la industria. Lo mostrado a continuación, es una imagen de cada uno de los estados de The Atlantic 50 marca con una empresa que no sólo es de confianza sino que también ha establecido su sede oficial en ese estado. Sin embargo, lo que no está demostrado es que está impulsando el compromiso.

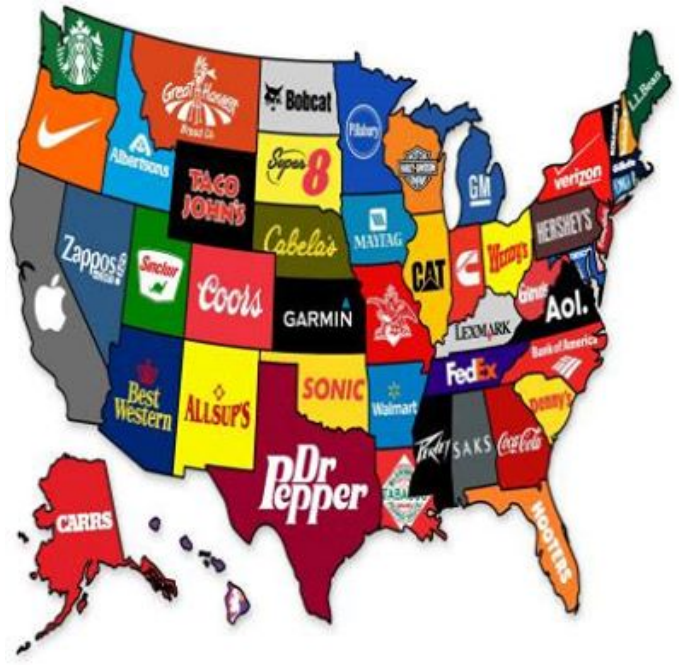

Imagen1. The Atlantic 50 marca Fuente:(InfiniGraph, 2013)

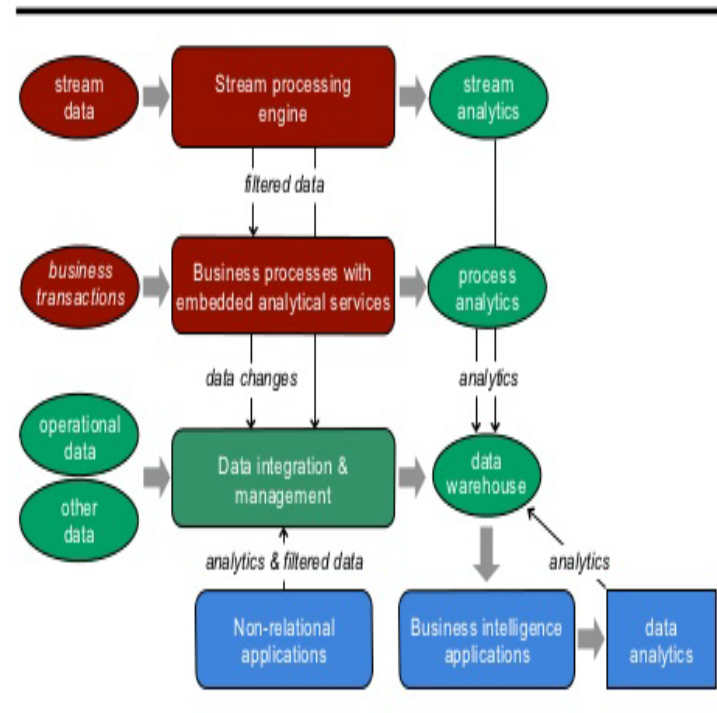

Imagen 2. Integración de procesos Fuente:(White, 2011)

El futuro Ilega al Business Intelligence: Nada mejor que empezar con hechos y datos. La prestigiosa compañía de estudios IDC en su reciente informe: Worldwide Business Analytics Software 2012-2016 Forecast and 2011 Vendor Shares, indica 
que el Business Intelligence va a crecer a un ritmo de un 9,8\% anual. Este dato es, sin lugar a dudas, uno de los más importantes a la hora de abordar el futuro del Business Intelligence. Nos encontramos con un sector en crecimiento, cercano a los dos dígitos, lo cual no es algo habitual en los tiempos actuales y con una continuación prevista en el tiempo.(Stratebi, 2013)

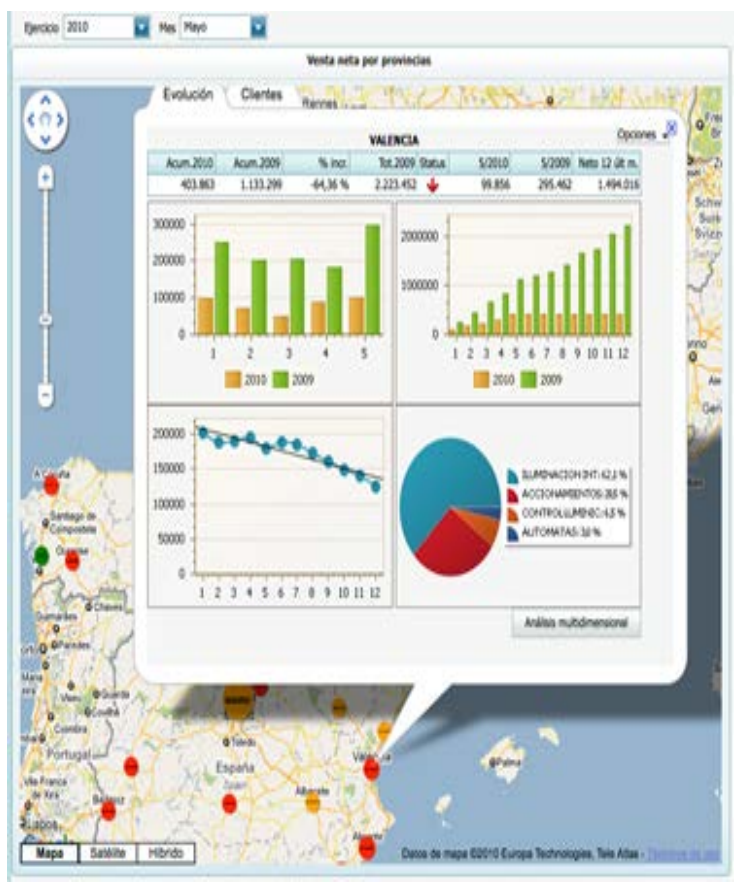

Imagen 3. Ejemplo de análisis -BI Fuente:(Urquizu, n.d.)

\section{Resultados}

Se vislumbra un potencial y atractivo uso de las redes sociales en la actualidad. Se puede evidenciar claramente que la red social más utilizada en la actualidad es la red social Facebook 750000000 visitantes , la misma que nos puede ayudar en los negocios inteligentes, que mediante un análisis de los datos almacenados en esta red social puedo seleccionar las diferentes características que puedan ayudar a mi empresa e invertir en esta red social para atraer potenciales nuevos clientes, así también podemos ver que twitter es una buena opción para invertir y acercar nuestra empresa a los futuros clientes de nuestra empresa o negocio.

\section{Conclusiones}

Actualmente y en un mundo globalizado todos los días se publica nueva información en las redes sociales, para que las empresas puedan tener una mejor inteligencia de negocios sobre lo que sirve y lo puedan utilizar en su beneficio para desarrollar mejor su estrategia, deben de utilizar herramientas como InfiniGraph, para poder tomar control de las billones de publicaciones que existen semanalmente.

Desarrollar una estrategia basada en lo que ya es tendencia y es viral, le resultara más fácil que tratar de desarrollar una estrategia basada en simples suposiciones.

\section{Referencias Bibliografía}

[1] Bidart, C. (2009). En busca del contenido de las redes sociales: los motivos de las relaciones. ... : Revista Hispana Para El Análisis de Redes Sociales, 6, 7. Retrieved from http://dialnet.unirioja.es/servlet/articulo ?codigo $=3041699$

[2] Breve manual de redes sociales para Pymes - Forbes México. (n.d.).

[3] Caldevilla, D. (2010). Las Redes Sociales. Tipología, uso y consumo de las redes 2.0 en la sociedad digital actual. Documentación de Las Ciencias de La Información, 33, 4568.

[4] Caldevilla Domínguez, D. (2010). Las Redes Sociales . sociedad digital actual. Documentación de Las Ciencias de La Información, 33(33), 45-68. Retrieved from http://dialnet.unirioja.es/servlet/articulo ?codigo $=3250105 \&$ info=resumen

[5] InfiniGraph. (2013). Mega Brand Content Marketing and Disruptive Effect of SEO | InfiniGraph. Retrieved February 16, 2014, from http://blog.infinigraph.com/2013/08/19/ mega-brand-content-marketing-anddisruptive-effect-of-seo/

[6] Internet, F. e. (2013). MARKETING EN REDES SOCIALES, INTERNET Y FACEBOOK. Retrieved February 13, 2014, from

http://facebookmicroweb.com/precioscotizacion-redes-sociales-publicidadmarketing-microweb-disenowebmarca-social-negocio-online/ 
[7] Neuman, G. (2013). Inteligencia de negocios, en qué ayuda a tu negocio y cuál es su proceso | GestioPolis. Retrieved from http://www.gestiopolis.com/canales2/g erencia/1/busint.htm

[8] Opinión: El uso de las redes sociales en el trabajo | Crhoy.com. (n.d.).

[9] Richard Ramírez-Anormaliza1, Xavier Linàs-Audet2, F. S. (2013).

Evaluación de los Sistemas ELearning: Estudio de Las Publicaciones Realizadas En La Web Of Knowledge, 13.

[10] Stratebi. (2013). Propuesta Gnarum Nuevas_Tendencias_BI.pdf. Retrieved February 16, 2014, from http://www.stratebi.es/todobi/May13/N uevas_Tendencias_BI.pdf

[11] Urquizu, P. (n.d.). Artículos Business Intelligence sobre Cuadros de mando. Retrieved October 20, 2014, from http://www.businessintelligence.info/c uadros-de-mando.html

[12] White, C. (2011). Using Big Data for Smarter Decision Making. BI Research, (July), 1-11. Retrieved from http://www.ithound.com/download/sm arter-decision-9129 